\title{
Bacteraemia following orotracheal intubation and oesophageal balloon dilatation
}

\section{CARSTEN PALNÆS HANSEN, HENRIK WESTH, KARL-ERIK BROK, RENE JENSEN, SVEND BERTELSEN}

From the Departments of Thoracic and Vascular Surgery, Clinical Microbiology, and Diagnostic Radiolog Bispebjerg Hospital, Copenhagen, Denmark

ABSTRACT Asymptomatic bacteraemia following balloon dilatation was assessed in 20 adults with oesophageal stricture. Asymptomatic bacteraemia occurred in 12 of 19 patients. The source of the bacteraemia appeared to be the patients' oropharyngeal flora. The bacteraemia was not of clinical importance in our patients, but might lead to endocarditis in predisposed individuals.

Many dental, gastroenterological, and urological procedures are associated with transient bacteraemia, which is often asymptomatic. ${ }^{\prime}$ Endocarditis is predominantly caused by streptococci of the oral flora, so prophylaxis must focus on dental and surgical procedures affecting the mouth, nasal cavity, throat, and oesophagus. The incidence of bacteraemia associated with oesophageal dilatation with bougies has varied in reports from zero to $100 \% .^{2-4}$ This study was undertaken to investigate whether bacteraemia occurred after oesophageal balloon dilatation.

\section{Methods}

We studied 20 patients with a median age of 71 (range 47-86) years. None had received an antibiotic during the week before dilatation. Sixteen patients had a peptic stricture of their distal oesophagus and four patients stenosis of an oesophagogastrotomia following surgery for carcinoma of the oesophagus.

Dilatations were performed with a $6 \mathrm{~cm}$ long balloon at the end of a double lumen catheter (William Cook, Europe APS, Soeborg, Denmark). A guide wire is passed to facilitate introduction of the catheter through narrow strictures. Through the second lumen the balloon can be inflated to either 15 or $22 \mathrm{~mm}$ (two models). After general anaesthesia and orotracheal intubation the catheter was passed orally and under fluoroscopic control through the stricture. After partial inflation the balloon was withdrawn into the stricture and inflated to full diameter. After 10 minutes the balloon was deflated and withdrawn. The balloons were reused two to four times after disinfection and venting.

Blood samples were drawn from the antecubital vein after iodine disinfection before and after intubation and after oesophageal dilatation. Aliquots of $10 \mathrm{ml}$ of each $20 \mathrm{ml}$ sample of blood were inoculated into two Roche blood

Address for reprint requests: Dr $\mathbf{H}$ Westh, Department of Clinical Microbiology, Bispebjerg Hospital, DK-2400 Copenhagen NV, Denmark.

Accepted 10 April 1989 culture bottles. They were cultured aerobically anif anaerobically and observed over six days for growth. A bacteria were identified to the genus level and most to the species level. Streptococci were examined by the streptococt cus department (Dr J Henrichsen) of the Statens Serumid stitut, Copenhagen, Denmark. ${ }^{5}$ Informed consent wog obtained from all patients; the study received the approval of our regional ethics committee.

The statistical methods used were Fisher's exact test f $\overrightarrow{q r}$ fourfold tables and 95\% "exact" confidence limits for values.

\section{Results}

Patient 1 inadvertently had the dilatation while he had pyelonephritis (an infection with Proteus mirabilis). All the other patients had an uneventful dilatation. All bloes cultures before dilatation were negative except for those patient 1 ( $P$ mirabilis cultured on all occasions). A positiole blood culture was obtained after orotracheal intubation three of the 20 patients and after oesophageal dilatation in (table). The positive blood cultures in patients 14 and 15 ape considered to be due to skin flora contaminants because both grew Propionibacterium acnes.

Four disinfected balloons were tested for contaminants. $\overline{\mathscr{P}}$ two cases there was no growth and in two there was less tham

Bacteriological findings in blood cultures from 20 patients having balloon dilatation of the oesophagus

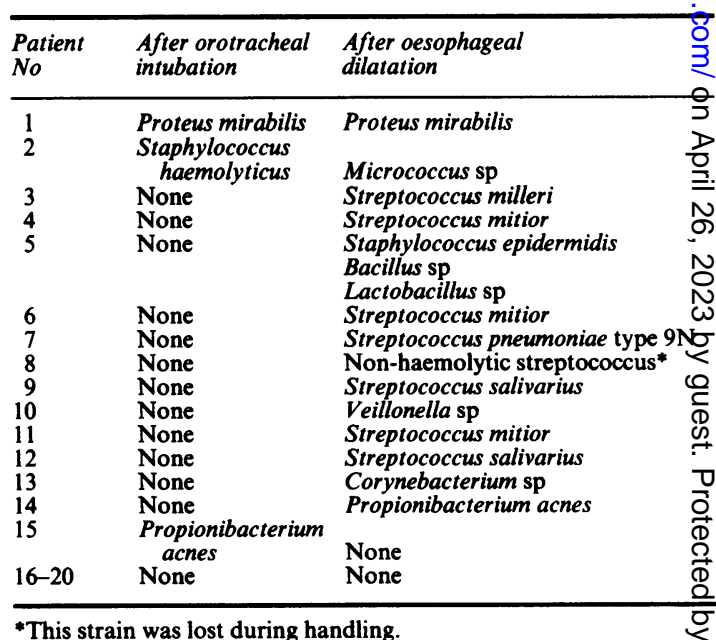


0.5 colony forming unit $/ \mathrm{cm}^{2}$ balloon, representing four different coagulase negative staphylococci.

\section{Discussion}

In this study 12 positive blood cultures out of 19 (excluding the patient with pyelonephritis) are presumed to be due to the oesophageal dilatation. The bacteria cultured are all thought to originate from the patients' oropharyngeal flora.

Blood was present on the balloon in 13 cases after dilatation; four patients had been operated on for cancer and three patients were receiving an $\mathrm{H}_{2}$ blocker. None of these observations was related to the occurrence of bacteraemia.

Bacteraemia occurs less frequently after orotracheal than after nasotracheal intubation-in one study zero versus $16 \% .^{6}$ This is in accordance with our finding of one in 18 cases. In studies of bacteraemia after oesophageal dilation with bougies the investigators have discussed whether their findings were due to oral flora or contaminated bougies. ${ }^{2-4}$ The types of oral bacteria isolated from our patients do not support contamination of the utensils as the cause of the bacteraemia arising from oesophageal dilatation. ${ }^{2}$ The isolation of coagulase negative staphylococci from two of the four balloons was probably due to inappropriate handling after disinfection and we no longer reuse our balloons.

The oesophagus will always be colonised with oropharyngeal flora, and the bougie or balloon cannot be positioned without surface contamination with these bacteria. Dilata- tion will often produce mucosal injury and the possibility of bacteraemia. The bacteraemia will usually be of no importance, but the risk of endocarditis in predisposed individuals and of septicaemia in the immunocompromised patient must be born in mind. These patients should be given prophylactic antibiotics, preferably penicillin $G$ intravenously, before oesophageal dilatation.

\section{References}

1 Shorvon PJ, Eykyn SJ, Cotton PB. Gastrointestinal instrumentation, bacteraemia, and endocarditis. Gut 1983;24:1078-93.

2 Raines DR, Branche C, Anderson DL, Boyce HW Jr. The occurrence of bacteremia after esophageal dilation. Gastrointestinal Endoscopy 1975;22:86-7.

3 Stephenson PM, Dorrington L, Harris OD, Rao O. Bacteraemia following oesophageal dilatation and oesophago-gastroscopy. Aust NZ Med 1977;7:32-5.

4 Welsh JD, Griffiths WJ, McKee RN, Wilkonson D, Flournoy DJ, Mohr JA. Bacteremia associated with esophageal dilatation. $J$ Clin Gastroenterol 1983;5:109-12.

5 Perch B, Kjems E, Ravn T. Biochemical and serological properties of Streptococcus mutans from various human and animal sources. Acta Pathol Microbiol Scand Sect B 1974;82:357-70.

6 Berry FA Jr, Blankenbaker WL, Ball CG. A comparison of bacteremia occurring with nasotracheal and orotracheal intubation. Anesth Analg 1973;52:873-6. 